\title{
Identification of prognostic long intergenic non-coding RNAs as competing endogenous RNAs with KRAS mutations in colorectal cancer
}

\author{
JUN XU ${ }^{1}$, QIU-YUN HUANG ${ }^{1}$ and CUN-JIN GE ${ }^{2}$ \\ Departments of ${ }^{1}$ General Surgery and ${ }^{2}$ Gastroenterology, The First College of Clinical Medical Science, \\ China Three Gorges University, Yichang, Hubei 443000, P.R. China
}

Received February 2, 2021; Accepted May 28, 2021

DOI: 10.3892/ol.2021.12978

\begin{abstract}
Colorectal cancer (CRC) is recognized as a common type of human cancer, and KRAS mutations are correlated with poor CRC survival outcomes. The evaluation and prediction of CRC results remain challenging. In the present study, RNA sequencing and clinical data from The Cancer Genome Atlas were used to identify KRAS mutation-related prognostic long intergenic non-coding RNAs (lincRNAs) in CRC. Significantly dysregulated lincRNAs and independent prognostic lincRNAs with KRAS mutations in CRC were identified. Two lincRNAs with KRAS mutations, LINC00265 and AL390719.2, were selected as key prognostic lincRNAs for both 10- and 5-year survival rates. In addition, competing endogenous (ce)RNA models were constructed to comprehensively assess the oncogenic performance of the two key lincRNAs. The ceRNA models suggested that LINC00265 and AL390719.2 are critical for the cell cycle and cancer pathways. Finally, reverse transcription-quantitative PCR was used to validate the ceRNA models in 12 pairs of CRC tissue samples. These prognostic lincRNAs may provide novel biomarkers for the prognostic prediction of CRC. The ceRNA model may also demonstrate the underlying mechanism of these lincRNAs in CRC.
\end{abstract}

\section{Introduction}

Colorectal cancer (CRC) is a common malignant tumor in the gastrointestinal tract; its morbidity and fatality rates are behind only gastric, esophageal and primary liver cancer in malignant tumors of the digestive system $(1,2)$. Globally, the incidence and mortality of CRC rank fifth among all malignant tumors (3). The

Correspondence to: Mrs. Qiu-Yun Huang, Department of General Surgery, The First College of Clinical Medical Science, China Three Gorges University, 183 Yiling Street, Wujiagang, Yichang, Hubei 443000, P.R. China

E-mail: 630655667@qq.com

Key words: colorectal cancer, long intergenic non-coding RNA, KRAS mutation, microRNAs incidence of $\mathrm{CRC}$ is associated with factors such as age, region and sex. CRC primarily occurs in middle-aged and elderly people ( $>40$ years old), and the incidence of CRC in men and women is relatively similar (4). Previously, the incidence and mortality rates of CRC have demonstrated a notable upward trend (5). Numerous studies have identified KRAS mutations as poor prognostic biomarkers correlated with poor CRC survival outcomes $(6,7)$. Taking advantage of synthetic lethal interactions with KRAS mutation may represent a target for effective therapeutic strategies in patients with KRAS-mutant CRC (8). Thus, detection methodology should be used to identify the key prognostic biomarkers for CRC, specifically KRAS-mutant CRC.

Human coding genes account for $<2 \%$ of the total genome. The number of transcripts in the genome is very large; $70 \%$ of the whole human genome is stably transcribed into RNAs, and the majority of these are non-coding RNAs (9), including microRNAs (miRNAs/miRs) and long non-coding RNAs (lncRNAs). IncRNAs refer to transcripts $>200$ nucleotides in length that do not contain a protein-coding sequence (10). Long intergenic non-coding RNAs (lincRNAs) are the largest class of lncRNA molecules (11). lincRNAs can serve as transcriptional regulators and influence gene transcription, acting as decoys to bind proteins or miRNAs $(9,12)$. Previously, numerous studies have reported that lincRNAs serve tumor-suppressive or tumor-promoting roles. For instance, lincRNAs correlated with CRC include CCAT1, CCAT2, CRNDE, HULC and MALAT1 $(13,14)$.

In the past several years, certain studies have reported crosstalk between lincRNAs and miRNAs during cancer progression, specifically the hypothesis of competing endogenous RNAs (ceRNAs) $(14,15)$. The ceRNA hypothesis model states that all coding and non-coding RNAs sharing common miRNA response elements may inhibit and indirectly regulate the expression of each other by competing for miRNA binding sites (16). These models are critical for human cancer. For instance, CCAT1 epigenetically downregulates c-Myc by serving as a ceRNA for miR-155, which downregulates c-Myc expression (16). lincRNAs are included in the regulatory network of CLDN4 via ceRNA-mediated miRNA evasion in gastric cancer (17). Thus, ceRNAs comprising lincRNAs, miRNAs and mRNAs can serve as important prognostic biomarkers in human cancer. 
Targeting KRAS-driven cancer is an effective strategy that selectively inhibits cancer growth while unharmed normal cells (18). Thus, it is necessary to explore and identify key lincRNAs in KRAS-mutant CRC. However, there appear to be few related previous studies. In the present study, RNA sequencing (RNA-Seq) and clinical data from The Cancer Genome Atlas (TCGA) were employed to identify key survival lincRNAs associated with KRAS mutations in CRC. Furthermore, RNA-Seq and miRNA-Seq data from TCGA were used to construct ceRNA models among lincRNAs, miRNAs and mRNAs in CRC. The current study may provide a new understanding of KRAS-mutated CRC and help to interpret the mechanisms underlying the functions of these lincRNAs in CRC.

\section{Materials and methods}

Raw data. RNA-Seq, miRNA-Seq, copy number variation (CNV) and clinical data of CRC (COAD + READ) were downloaded from the TCGA (https://portal.gdc.cancer.gov). The RNA-Seq data are presented as fragments per kilobase million (FPKM). The miRNA-seq data were presented as reads per million miRNA mapped data.

Dysregulated lincRNA analysis. Dysregulated gene analysis was conducted with $\mathrm{R}$ software version 4.0.3, and the Mann-Whitney $U$ test was performed to define significantly dysregulated lincRNAs between tumor and normal samples. To decrease background noise, any lincRNAs with 50 th percentile FPKM=0 in tumor or normal samples were eliminated. Significantly dysregulated lincRNAs were defined as a logarithmic transformed fold-change $\left(\left|\log _{2}(\mathrm{FC})\right|\right) \geq 1$ and a false discovery rate (FDR) $\leq 0.05$. Furthermore, lincRNAs with a mean FPMK value $>1$ in tumor samples were considered upregulated, and lincRNAs with a mean FPMK value $>1$ in normal samples were considered downregulated.

Predictive value of lincRNAs for survival in patients with KRAS mutations. Receiver operating characteristic (ROC) curves were used to analyze the association between lincRNAs and the survival status of patients with mutant or wild-type KRAS at 10 and 5 years. ROC curves were determined to evaluate the sensitivity and specificity of the expression level of each lincRNA in predicting mortality in patients (19). Forest plots were used to demonstrate the result of ROC. An area under the curve $>0.6$ and a $\mathrm{P}<0.05$ were the threshold values to indicate significance.

Identification of key survival-related lincRNAs with KRAS mutations. Robust likelihood-based survival models were constructed to identify the key lincRNAs influencing the prognosis of CRC using rbsurv (https://bioconductor.org/packages/rbsurv) in $\mathrm{R}$ software version 4.0.3. The lincRNAs with the highest frequency were selected as the final feature lincRNAs. The effect of key lincRNA expression on patient survival was assessed by the Kaplan-Meier survival curves and the log-rank test.

Prediction of target lincRNAs and mRNAs of miRNAs. Target lincRNAs and mRNAs were predicted for miRNAs using the online tool DIANA (http://diana.imis.athena-innovation. gr/DianaTools/index.php?r=tarbase/index). The cut-off values used to identify a significant correlation were
Pearson correlation coefficient $(\mathrm{PCC})$ of $<-0.1$ and $\mathrm{P}<0.05$. Furthermore, a maximal information coefficient (MIC) $>0.17$ was set as a cut off for significant correlation (20). PCC and MIC were performed with Rsoftware version 4.0.3.

Functional enrichment analysis. Functional enrichment analysis was performed with Kyoto Encyclopedia of Genes and Genomes (KEGG), Gene Ontology (GO) and Reactome under Metascape (http://metascape.org) (21). $\mathrm{P}<0.05$ was set as the cut-toff.

Regulatory network. Regulatory network lincRNAs were directly connected to miRNAs, and mRNAs were directly connected to the miRNAs, constituting a regulatory network. The network was constructed and displayed using Cytoscape 3.7.2 (https://cytoscape.org).

Reverse transcription-quantitative $(R T-q) P C R$. A total of 12 pairs of CRC tissues and normal tissues (normal tissues were $2-3 \mathrm{~cm}$ away from cancer tissue) were obtained from 12 patients (age range, 42-53 years; median age, 48 years; eight men and four women) who underwent radical resection at The First College of Clinical Medical Science, China Three Gorges University (Yichang, China) between July 2020 and September 2020. The patient was diagnosed as CRC by imaging and pathological examination, and did not receive chemotherapy or radiotherapy before operation. All samples are stored in $-80^{\circ} \mathrm{C}$ refrigerator for use. Total RNA was extracted using TRIzol ${ }^{\circledR}$ reagent (Invitrogen; Thermo Fisher Scientific, Inc.). RNA was reverse transcribed into cDNA using the PrimeScript 1st Strand cDNA Synthesis Kit Starter kit (Takara Bio, Inc.), according to the manufacturer's protocol. Relative expression levels of lincRNAs and mRNAs were quantified using the TB Green ${ }^{\circledR}$ Premix Ex Taq ${ }^{\mathrm{TM}}$ II kit (Takara Bio, Inc.), according to the manufacturer's protocol. Relative expression levels of miRNAs were quantified using the Mir-X miRNA First-Strand Synthesis kit (Takara Bio, Inc.), according to the manufacturer's protocol. The primer sequences are listed in Table SI. Expression levels of lincRNAs and mRNAs relative to $\beta$-actin and expression of miRNAs relative to U6 snRNA were determined using the $2^{-\Delta \Delta \mathrm{Cq}}$ method (22). All experimental procedures were approved by the Ethics Committee of The First College of Clinical Medical Science, China Three Gorges University. Written informed consent was provided by all patients prior to the study.

Statistical analysis. The Mann-Whitney U test was performed using GraphPad Prism (GraphPad Software, Inc.) version 9 software and R software version 4.0.3. Kaplan-Meier survival curves and log-rank tests were used to evaluate the effect of lincRNA expression on survival. Receiver operating characteristic (ROC) curves were generated using GraphPad Prism version 9 software. Data are presented as the mean \pm SEM. $\mathrm{P}<0.05$ was considered to indicate a statistically significant difference.

\section{Results}

Identification of significantly dysregulated lincRNAs in CRC. To identify the dysregulated lincRNAs in CRC, RNA-Seq data from 647 tumor and 51 normal (including 50 pairs) 
A



B Tumor $(\mathrm{N}=50)$ Normal $(\mathrm{N}=50)$

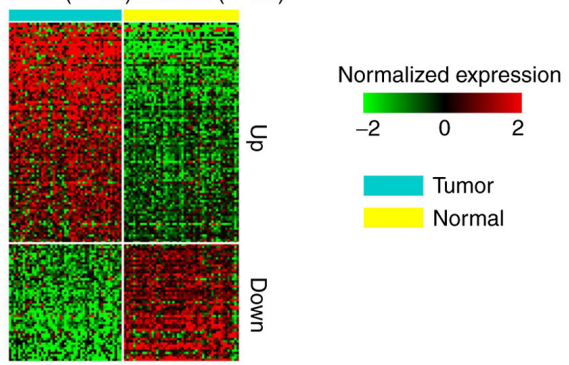

Figure 1. Dysregulated lincRNAs in CRC. (A) Heatmap displaying 147 dysregulated lincRNAs, using total tumor ( $\mathrm{n}=625$ ) and normal (n=51) CRC data from The Cancer Genome Atlas. (B) Heatmap displaying 147 dysregulated lincRNAs, using adjacent samples tumor ( $\mathrm{n}=50$ ) and normal ( $\mathrm{n}=50$ ) CRC data from The Cancer Genome Atlas. Each row represents a lincRNA and each column represents a sample. Each cell represents expression. Red represents high expression, while green represents low expression. Blue samples represent tumor and yellow samples represent normal. CRC, colorectal cancer; lincRNA, long intergenic non-coding RNA.
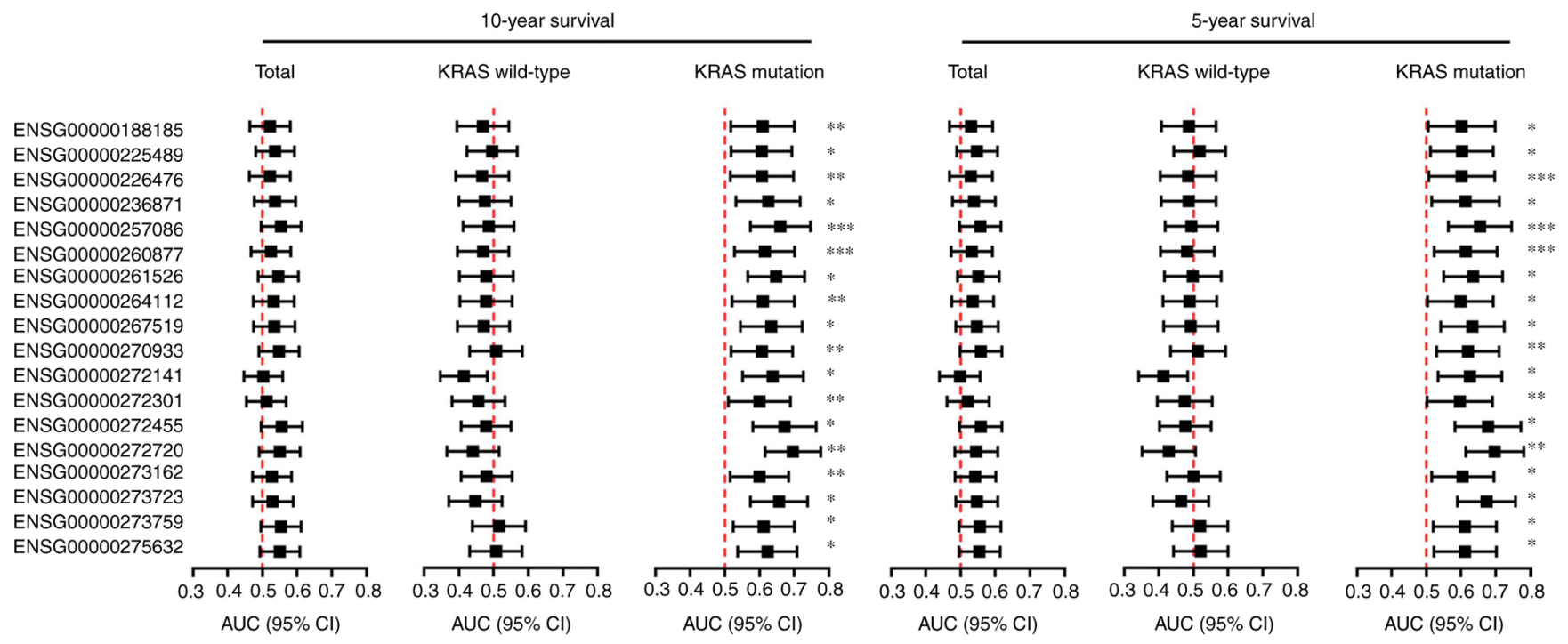

Figure 2. Independent prognostic lincRNAs with KRAS-mutant CRC. Forest plots displaying the AUCs and $95 \%$ CIs of 18 lincRNAs with regard to 10 - and 5-year survival in patients with CRC with mutant or wild-type KRAS. Each row represents a lincRNA, and the abscissa coordinate represents the AUC value. ${ }^{*} \mathrm{P}<0.05,{ }^{* *} \mathrm{P}<0.01,{ }^{* * * *} \mathrm{P}<0.001$ (death vs. alive). CRC, colorectal cancer; lincRNA, long intergenic non-coding RNA; AUC, area under curve; CI, confidence interval.

CRC samples from TCGA datasets were analyzed. $\log _{2}(\mathrm{FC})$ of lincRNA expression in tumor vs. normal $>1$ was classified as an upregulated gene, while $<-1$ was classified as a downregulated gene. In total, 7,369 lincRNAs were identified from the datasets. Subsequently, 96 upregulated lincRNAs and 51 downregulated lincRNAs were identified. Notably, these lincRNAs were also dysregulated in 50 tumor and adjacent normal samples (Fig. 1). These 147 lincRNAs were subsequently used to identify significant prognostic predictions.

Identification of independent prognostic lincRNAs with KRAS-mutant CRC. Patient 5- or 10-year survival rates are commonly used to represent statistical cure rates for those with cancer (23). Among the 647 tumor samples, 586 were identified as having 10 -year survival data, and 545 were identified as having 5-year survival data. To determine whether the 147 dysregulated lincRNAs influencing the survival of patients with CRC depended on KRAS mutations, 217 and 198 samples were identified as having a KRAS mutation at 10 and 5 years, respectively. Using ROC curve analyses, 18 lincRNAs were identified as independent prognostic markers in mutant KRAS, rather than wild-type KRAS clinical samples (Fig. 2). These 18 lincRNAs were used to identify key lincRNAs with KRAS mutations. 
Table I. Identification of key survival lincRNAs with KRAS mutations in 10-year survival.

\begin{tabular}{lccc}
\hline Gene stable ID & Symbols & Nloglik & AIC \\
\hline ENSG00000226476 & LINC01748 & 227.81 & 455.62 \\
ENSG00000188185 & LINC00265 & 224.88 & $451.76^{a}$ \\
ENSG00000272141 & AL390719.2 & 221.27 & $446.54^{a}$ \\
ENSG00000275632 & AL035461.2 & 221.01 & 448.03 \\
ENSG00000273759 & AL117379.1 & 220.85 & 449.70 \\
ENSG00000261526 & AC012615.1 & 219.33 & 450.65 \\
ENSG00000267519 & AC020916.1 & 219.31 & 448.62 \\
ENSG00000272455 & AL391244.3 & 217.74 & 451.49 \\
ENSG00000272301 & AP002360.3 & 217.73 & 449.45 \\
ENSG00000270933 & AC010719.1 & 217.70 & 453.41 \\
ENSG00000264112 & AC015813.1 & 217.68 & 455.36 \\
ENSG00000236871 & LINC00106 & 213.99 & 451.98 \\
ENSG00000257086 & AP001453.4 & 213.99 & 449.98 \\
ENSG00000260877 & AP005233.2 & 212.96 & 451.93 \\
ENSG00000225489 & AL354707.1 & 211.69 & 451.37 \\
ENSG00000273162 & AL133215.2 & 211.31 & 452.62 \\
ENSG00000272720 & AL022322.1 & 211.30 & 454.60 \\
ENSG00000273723 & AL139089.1 & 210.64 & 455.28
\end{tabular}

${ }^{\mathrm{a}} \mathrm{P}<0.05$. lincRNA, long intergenic non-coding RNA; AIC, akaike information criterion.

Identification of key prognostic lincRNAs with KRAS mutations. Random data analysis was performed using robust likelihood-based modeling 1,000 times. Statistical frequency analysis of the significantly changed lincRNAs in KRAS-mutant samples suggested that all the selected lincRNAs had a high frequency. Two lincRNAs, LINC00265 (Gene Stable ID, ENSG00000188185) and AL390719.2 (Gene Stable ID, ENSG00000272141), were identified as key prognostic lincRNAs with KRAS mutations at both 10 years (Table I) and 5 years (Table II). LINC00106 was only significant in the 5-years survival group.

Oncogenicity of key prognostic lincRNAs with KRAS mutations. To examine whether the expression level of the key lincRNAs LINC00265 and AL390719.2 was correlated with a less favorable prognosis in CRC with KRAS mutations, 10- and 5-year overall survival (OS) rates were analyzed in the CRC TCGA dataset. To evaluate the clinical significance of the key lincRNAs LINC00265 and AL390719.2 in the survival of patients with CRC, as well as their associations with the KRAS mutation status, the prognostic significance of these lincRNAs was determined using TCGA datasets. The 10- and 5-year OS rates suggested that LINC00265 (Fig. 3A and B) and AL390719.2 (Fig. 3C and D) expression levels were associated with patient survival in CRC. High LINC00265 (Fig. 3A and B) and AL390719.2 (Fig. 3C and D) expression was significantly associated with less favorable survival in patients with CRC with mutant KRAS, but not in those with wild-type KRAS (Fig. 3). Hence, LINC00265 and AL390719.2 upregulation specifically predicts a poor
Table II. Identification of key survival lincRNAs with KRAS mutations in 5-year survival.

\begin{tabular}{llll}
\hline Gene stable ID & Symbols & Nloglik & AIC \\
\hline ENSG00000226476 & LINC01748 & 208.3 & 416.61 \\
ENSG00000188185 & LINC00265 & 204.93 & $411.85^{a}$ \\
ENSG00000236871 & LINC00106 & 201.40 & $406.80^{a}$ \\
ENSG00000272141 & AL390719.2 & 199.35 & $404.69^{a}$ \\
ENSG00000272720 & AL022322.1 & 198.76 & 405.52 \\
ENSG00000275632 & AL035461.2 & 198.65 & 407.29 \\
ENSG00000272301 & AP002360.3 & 198.24 & 408.49 \\
ENSG00000270933 & AC010719.1 & 197.64 & 411.29 \\
ENSG00000273759 & AL117379.1 & 197.64 & 409.29 \\
ENSG00000264112 & AC015813.1 & 197.36 & 412.73 \\
ENSG00000225489 & AL354707.1 & 196.60 & 413.20 \\
ENSG00000273723 & AL139089.1 & 196.59 & 415.17 \\
ENSG00000260877 & AP005233.2 & 192.71 & 409.42 \\
ENSG00000267519 & AC020916.1 & 192.12 & 410.25 \\
ENSG00000272455 & AL391244.3 & 191.65 & 411.29 \\
ENSG00000261526 & AC012615.1 & 191.49 & 412.99 \\
ENSG00000257086 & AP001453.4 & 188.47 & 408.94 \\
ENSG00000273162 & AL133215.2 & 188.44 & 410.88
\end{tabular}

${ }^{\mathrm{a}} \mathrm{P}<0.05$. lncRNA, long intergenic non-coding RNA; AIC, akaike information criterion.

prognosis and represents an independent prognostic marker in KRAS-mutant CRC.

To investigate the expression of the key lincRNAs LINC00265 and AL390719.2, the pan-cancer expression levels and CRC CNV profiles of these lincRNAs were analyzed in TCGA dataset. LINC00265 and AL390719.2 were identified as being expressed in cancer, including CRC (Fig. 4A and B). It was observed that LINC00265 CNV was amplified in 81.54\% of CRC samples (Fig. 4C). LINC00265 expression was significantly higher in $\mathrm{CRC}$ with $\mathrm{CNV}$ amplification, and expression showed a positive correlation with CNV amplification (Fig. 4D). AL390719.2 expression was also significantly higher in CRC with CNV amplification (Fig. 4E), but CNV amplification was detected in $32.03 \%$ of CRC samples, and AL390719.2 expression was not associated with CNV amplification (Fig. 4F).

Regulatory modules of key prognostic lincRNAs with KRAS mutations. miRNA-lincRNA and miRNA-mRNA interactions were identified using tools from DIANA. PCC and MIC were used to evaluate the correlation of these interactions. The downregulated miRNAs were identified between tumor and normal samples with a $\log _{2}(\mathrm{FC})$ value $\leq-1$ and FDR $<0.05$. Finally, 2 miRNAs and 288 mRNAs connected to AL390719.2 (Fig. 5A) and 6 miRNAs and 415 mRNAs connected to LINC00265 (Fig. 5B) were identified. Functional enrichment analysis revealed that the mRNAs in the regulatory modules may be critical for the cell cycle in CRC ('cell cycle' from Reactome and KEGG, 'regulation of cell cycle process' from GO and 'pathways in cancer' from KEGG; Fig. 5C). To 

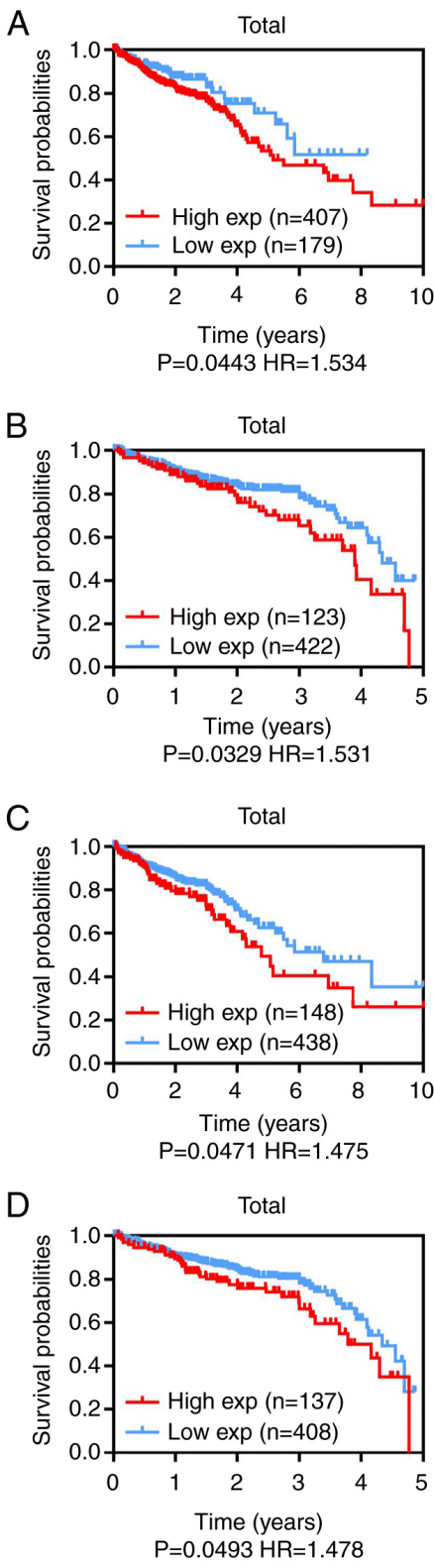
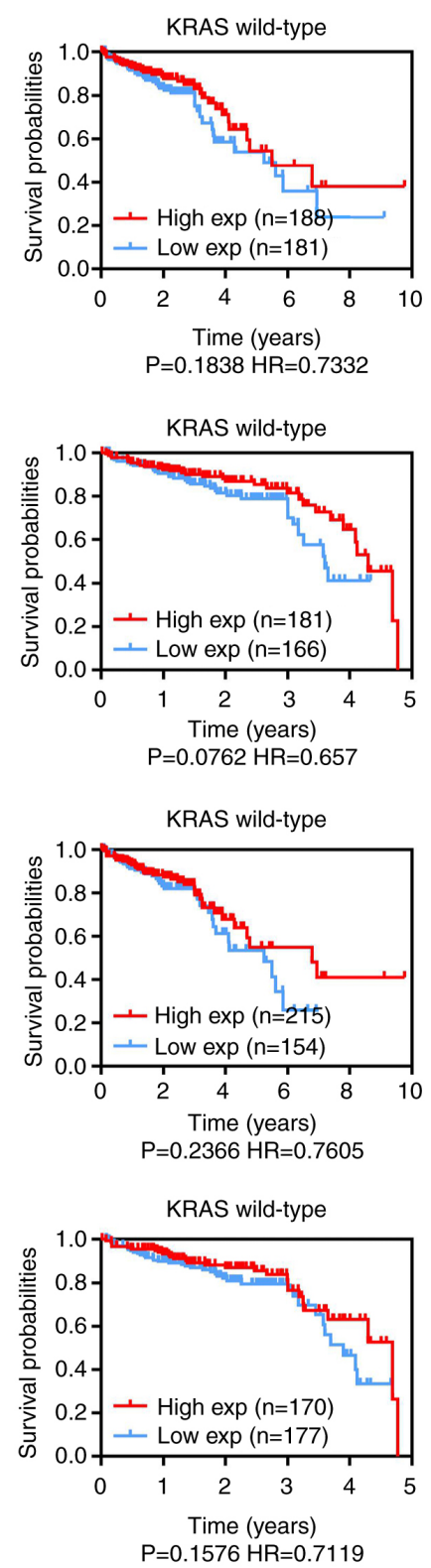
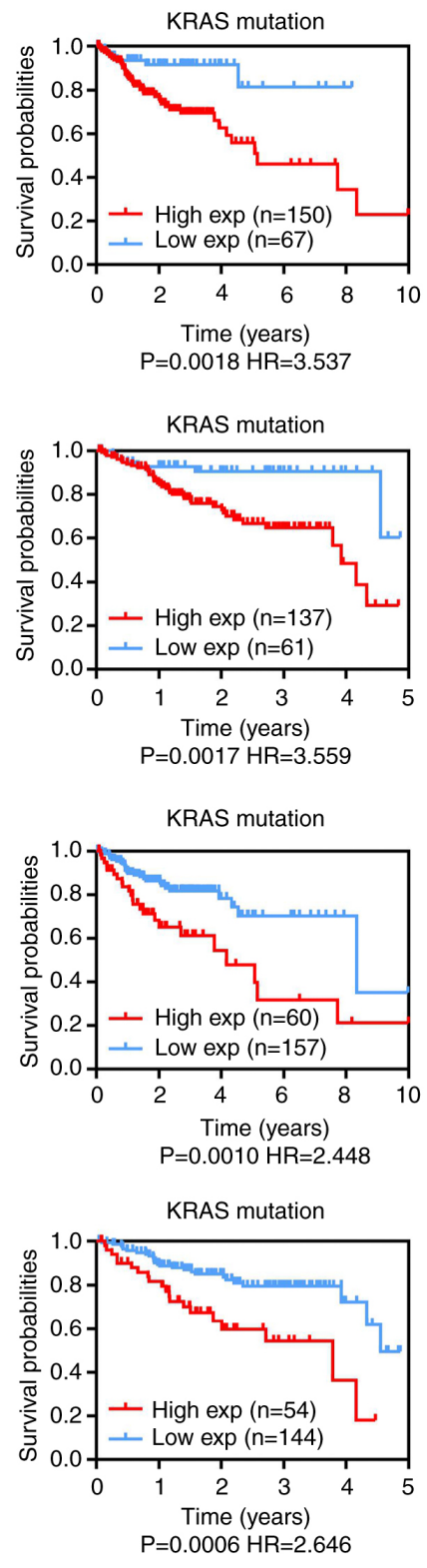

Figure 3. Key prognostic lincRNAs with KRAS mutations. (A-D) Kaplan-Meier survival curves for two key lincRNAs in total (left), KRAS wild-type (middle) and KRAS-mutant (right) CRC samples, for (A and B) LINC00265 and (C and D) AL390719.2. CRC, colorectal cancer; lincRNA, long intergenic non-coding RNA; HR, hazard ratio.

validate these findings, RT-qPCR was used to check lncRNAs, miRNAs and mRNAs ('hsa05200 pathways in cancer'; orange nodes in Fig. 5 network) in 12 pairs of CRC samples. RT-qPCR demonstrated that LINC00265, AL390719.2 and 12 genes were upregulated in tumor samples, while 2 miRNAs were downregulated in tumor samples (Fig. 6A and C). Furthermore, RT-qPCR demonstrated that these two miRNAs were negatively correlated with lncRNAs and mRNAs (Fig. 6B and D).

\section{Discussion}

CRC is now the third most common malignancy worldwide. Oncogenic KRAS mutations initiate and sustain CRC progression. Numerous studies have assessed KRAS mutations associated with CRC outcomes (24-26). To provide prognostic lincRNAs to predict the outcomes of patients with CRC with
KRAS mutations, 18 lincRNAs (LINC00265, AL390719.2, AL035461.2, AL117379.1, AC012615.1, AC020916.1, AL391244.3, AP002360.3, AC010719.1, AC015813.1, LINC00106, AP001453.4, AP005233.2, AL354707.1, AL133215.2, AL022322.1, AL139089.1 and LINC01748) were identified as independent prognostic lincRNAs in CRC with KRAS mutations. All these lincRNAs are upregulated in primary CRC tumors, and their increased expression is associated with a poor prognosis in patients with CRC with KRAS mutations. The expression levels of these lincRNAs were correlated with 5- and 10-year OS rates in patients with CRC. Furthermore, these 18 lincRNAs were independent prognostic markers in patients with CRC with mutant KRAS, but not in those with wild-type KRAS. The aforementioned results suggest that these lincRNAs may serve as prognostic biomarkers in CRC and correlate with CRC progression. 

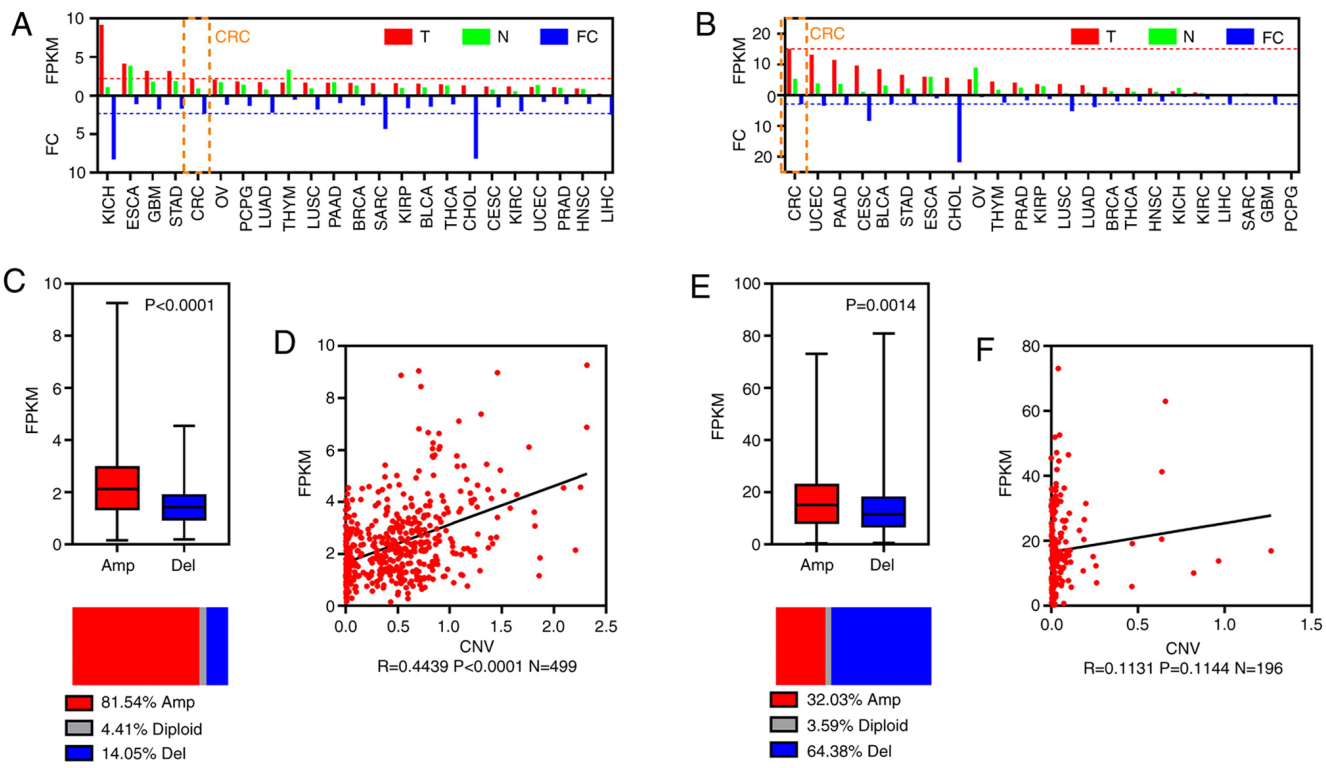

Figure 4. Oncogenicity of two key prognostic lincRNAs. Bar plots of FPKM and FC for (A) LINC00265 and (B) AL390719.2. (C) Boxplot showing FPKM (top) and horizontal slices showing the percentage group by CNV amplification and deletion of LINC00265. (D) Scatter diagram showing the correlation between CNV and FPKM for LINC00265. (E) Boxplot showing FPKM and horizontal slices showing the percentage group by CNV amplification and deletion of AL390719.2. (F) Scatter diagram showing the correlation between CNV and FPKM for AL390719.2. CRC, colorectal cancer; lincRNA, long intergenic non-coding RNA; FC, fold-change; FKPM, fragments per kilobase million; CNV, copy number variation; T, tumor; N, normal; Amp, amplification; Del, deletion; BLCA, bladder urothelial carcinoma, BRCA, breast invasive carcinoma, CESC, cervical squamous cell carcinoma and endocervical adenocarcinoma, CHOL, cholangiocarcinoma, CRC, colorectal cancer, ESCA, esophageal carcinoma, GBM, glioblastoma multiforme, HNSC, head and neck squamous cell carcinoma, KICH, kidney chromophobe, KIRC, kidney renal clear cell carcinoma, KIRP, kidney renal papillary cell carcinoma, LIHC, liver hepatocellular carcinoma, LUAD, lung adenocarcinoma, LUSC, lung squamous cell carcinoma, OV, ovarian serous cystadenocarcinoma, PAAD, pancreatic adenocarcinoma, PCPG, pheochromocytoma and paraganglioma, PRAD, prostate adenocarcinoma, SARC, sarcoma, STAD, stomach adenocarcinoma, THCA, thyroid carcinoma, THYM, thymoma, UCEC, uterine corpus endometrial carcinoma.

A
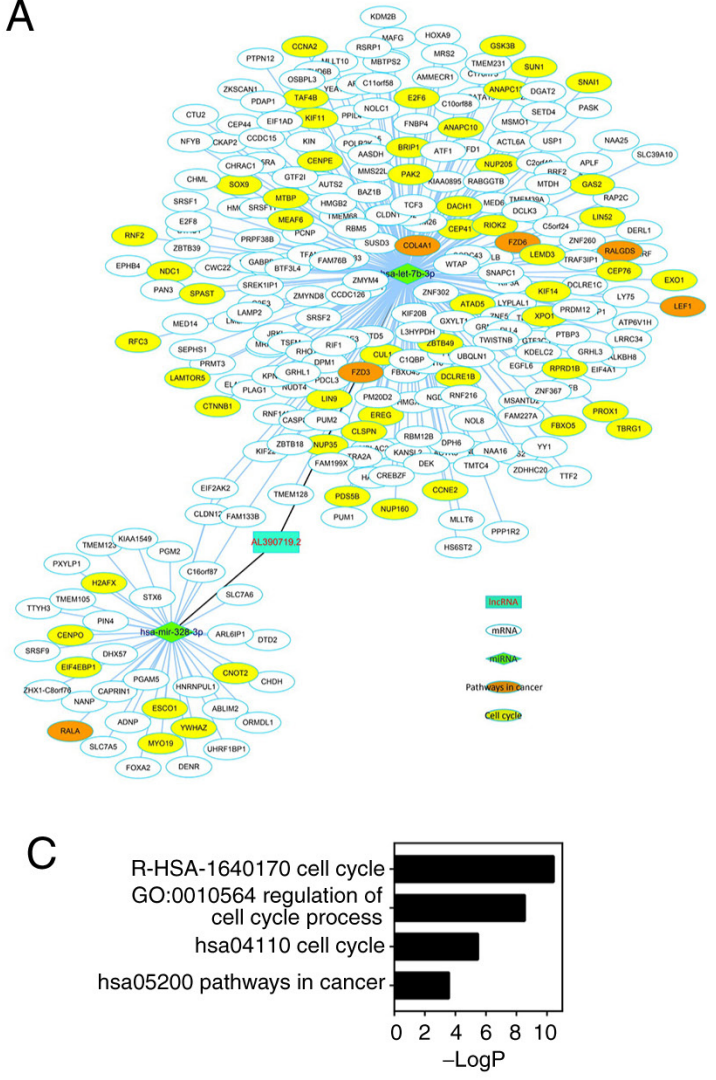

B

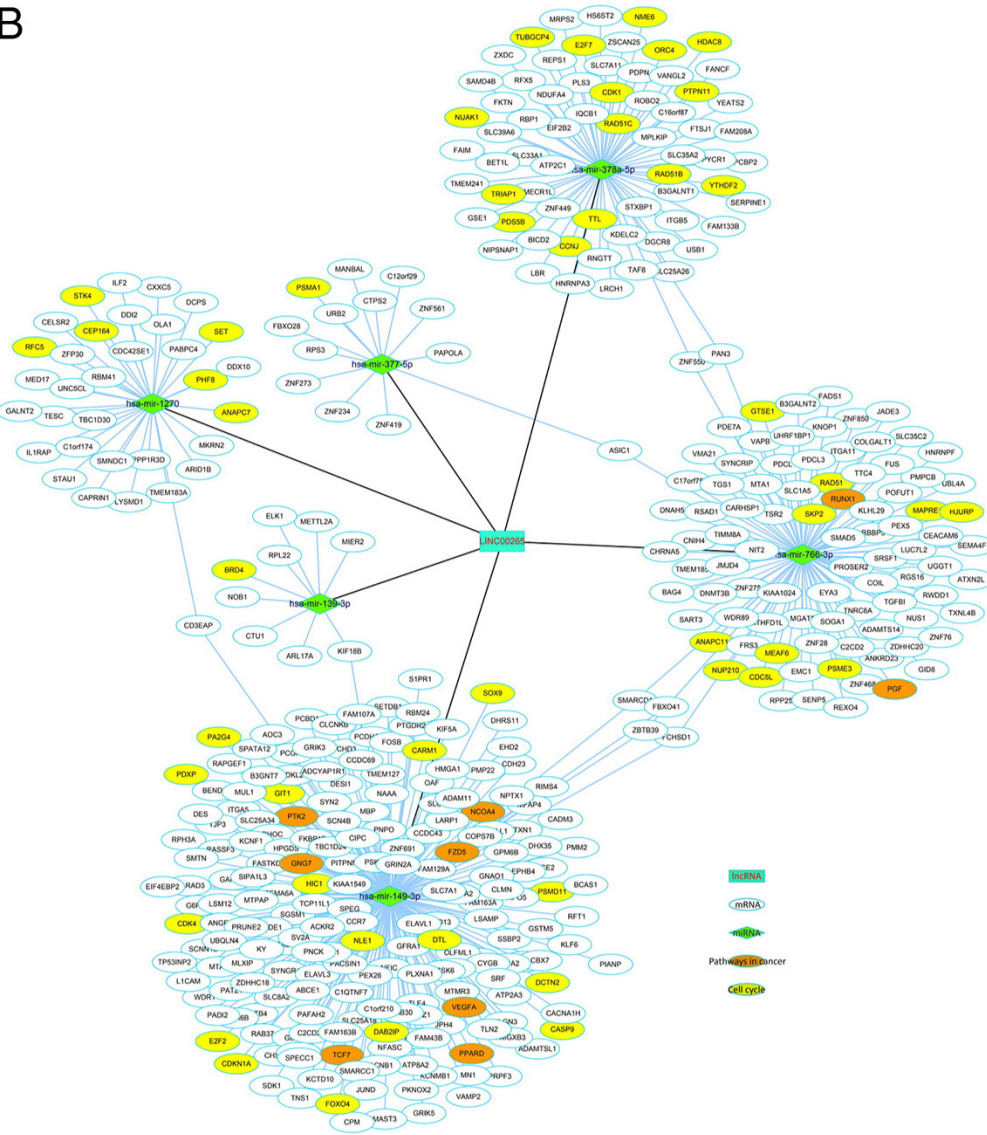

Figure 5. Regulatory modules of key prognostic lincRNAs. lincRNA-miRNA-mRNA network for (A) AL390719.2 and (B) LINC00265. (C) Bar plot of the cell cycle and cancer pathway for LINC00265- and AL390719.2-related mRNAs according to functional enrichment analysis. CRC, colorectal cancer; lincRNA, long intergenic non-coding RNA; GO, Gene Ontology; miRNA, microRNA. 
A
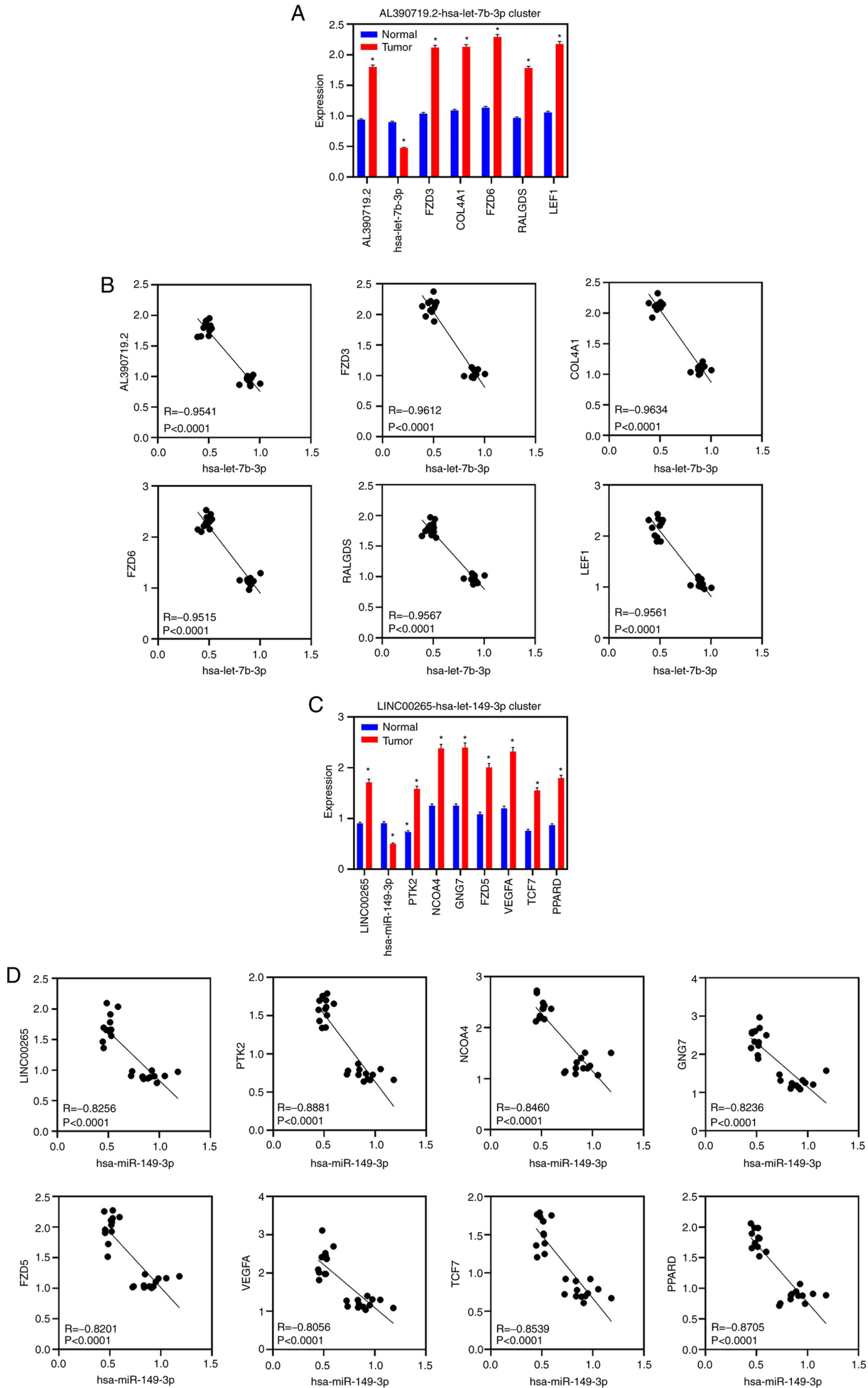

Figure 6. RT-qPCR validation in CRC samples. (A) RT-qPCR showing the expression of AL390719.2, hsa-let-7b-3p, FZD3, COL4A1, FZD6, RALGDS and LEF1 in 12 paired CRC and normal samples. Data are represented as the mean \pm SEM. (B) Scatter diagram showing the correlation between hsa-let-7b-3p and AL390719.2, FZD3, COL4A1, FZD6, RALGDS and LEF1 in 12 paired CRC and normal samples using RT-qPCR. (C) RT-qPCR showing the expression of LINC00265, hsa-mir-149-3p, PTK2, NCOA4, GNG7, FZD5, VEGFA, TCF7 and PPARD in 12 paired CRC and normal samples. Data are presented as the mean \pm SEM. (D) Scatter diagram showing the correlation between hsa-mir-149-3p and LINC00265, PTK2, NCOA4, GNG7, FZD5, VEGFA, TCF7 and PPARD in 12 paired CRC and normal samples by RT-qPCR. "P<0.05 (tumor vs. normal) CRC, colorectal cancer; lincRNA, long intergenic non-coding RNA; RT-qPCR, reverse transcription-quantitative PCR; miR, microRNA. 
Robust likelihood-based survival models were used to identify two key lincRNAs, LINC00265 and AL390719.2, from 18 lincRNAs. All 18 lincRNAs were first identified as dysregulated and correlated with poor prognosis with KRAS mutations in human cancer. LINC00265 was reported to be differentially expressed and revealed to be a prognostic biomarker in the lung adenocarcinoma (LUAD) TCGA dataset (27). LINC00265 was also demonstrated to be upregulated in LUAD samples from TCGA. It was revealed that LINC00265 expression in CRC tumors was significantly associated with CNV amplification. Notably, CNV amplification was reported as an upstream mechanism to increase gene expression $(28,29)$. The present results indicated that CNV amplification may cause LINC00265 overexpression in CRC. In addition, AL390719.2 was shown to be highly expressed in CRC tumors, but its increased expression was not caused by CNV amplification. Furthermore, Kaplan-Meier survival curves revealed that LINC00265 and AL390719.2 expression was associated with 5- and 10-year OS rates. High LINC00265 and AL390719.2 expression was correlated with less favorable 5- and 10-year OS rates in patients with mutant KRAS, but not in those with wild-type KRAS. Hence, LINC00265 and AL390719.2, as key prognostic lincRNAs in KRAS-mutant CRC, may be used to predict survival for patients with CRC.

To identify the function and molecular mechanism of LINC00265 and AL390719.2, it was determined whether these two key lincRNAs, as ceRNAs bound to miRNAs, regulate important genes. It was revealed that 2 miRNAs and 288 mRNAs were associated with AL390719.2, and that 6 miRNAs and 415 mRNAs were associated with LINC00265. Notably, functional enrichment analysis suggested that mRNAs in the regulatory modules were enriched in cell cycle biological processes. LINC00265 and AL390719.2 may serve as ceRNAs to bind these miRNAs and prevent the inhibitory effect of miRNAs on cell cycle genes, resulting in the upregulation of cell cycle genes and promoting CRC progression. AL390719.2, as a ceRNA, binds with hsa-mir-328-3p and hsa-let-7b-3p. LINC00265, as a ceRNA, binds to hsa-mir-1270, hsa-mir-139-3p, hsa-mir-149-3p, hsa-mir-377-5p, hsa-mir-378a-5p and hsa-mir-766-3p. These miRNAs were downregulated in CRC samples from TCGA. In addition, hsa-let-7b-3p, hsa-mir-328-3p, hsa-miR-139-3p, hsa-mir-149-3p and hsa-miR-378a-5p have been reported to be downregulated in CRC (30-34). Certain miRNAs have been reported to impact the cell cycle in human cancer. For example, hsa-let-7b has been reported to impact the cell cycle to inhibit prostate cancer cell proliferation in vitro (35). Moreover, hsa-mir-149 directly regulates the expression of cyclin-dependent kinase 4 (CDK4) cell lines, and hsa-mir-149 overexpression results in $\mathrm{G}_{0}-\mathrm{G}_{1}$ arrest and cell death in CRC (36). Also, CDK1 was the target gene of hsa-mir-378a-5p, and hsa-mir-378a-5p decreased CDK1 expression in hepatocytes (37). CDK1 is critical for regulating the $\mathrm{G}_{2}-\mathrm{M}$ transition during cell cycle progression (38). CDK4 controls cell cycle progression via pocket proteins and $\mathrm{E} 2 \mathrm{~F}$ transcription factors, and is correlated with cancer development and progression (39). Therefore, LINC00265 and AL390719.2 may serve as ceRNAs through competitive interactions with these miRNAs, resulting in the low expression of these miRNAs followed by upregulation of cell cycle genes in CRC. Moreover, RT-qPCR was used to validate LINC00265, AL390719.2 and related miRNAs and mRNAs in CRC samples.

In conclusion, 18 lincRNAs were identified that were upregulated and have potential as independent prognostic markers in CRC with KRAS mutations. From these lincRNAs, LINC00265 and AL390719.2 were identified as key lincRNAs that serve important roles in CRC progression, as ceRNAs for the regulation of lincRNA-miRNA-mRNA networks. The present findings may provide novel prognostic markers and therapeutic targets for CRC. However, the investigation of the regulatory models among these genes remains necessary.

\section{Acknowledgements}

Not applicable.

\section{Funding}

No funding was received.

\section{Availability of data and materials}

The datasets generated and analyzed during the current study are available in The Cancer Genome Atlas [https://portal.gdc. cancer.gov].

\section{Authors' contributions}

JX conceptualized the present study, designed the research and performed bioinformatics analysis. JX and QYH performed the experiments. JX and CJG analyzed and interpreted the data. JX drafted and edited the manuscript, and QYH supervised the project. JX, QY and CJG confirmed the authenticity of all the raw data. All authors have read and approved the final manuscript.

\section{Ethics approval and consent to participate}

The present study was approved by the Ethics Committee of The First College of Clinical Medical Science, China Three Gorges University (Yichang, China; approval no. HEC-KYJJ-2019-056-01). Written informed consent was provided by all patients prior to the study start.

\section{Patient consent for publication}

Not applicable.

\section{Competing interests}

The authors declare that they have no competing interests.

\section{References}

1. Nasseri Y and Langenfeld SJ: Imaging for colorectal cancer. Surg Clin North Am 97: 503-513, 2017.

2. Mármol I, Sánchez-de-Diego C, Pradilla Dieste A, Cerrada E and Rodriguez Yoldi MJ: Colorectal carcinoma: A general overview and future perspectives in colorectal cancer. Int J Mol Sci 18: 197,2017 
3. Bray F, Ferlay J, Soerjomataram I, Siegel RL, Torre LA and Jemal A: Global cancer statistics 2018: GLOBOCAN estimates of incidence and mortality worldwide for 36 cancers in 185 countries. CA Cancer J Clin 68: 394-424, 2018.

4. Patel SG and Ahnen DJ: Colorectal cancer in the young. Curr Gastroenterol Rep 20: 15, 2018.

5. Siegel RL, Miller KD, Fedewa SA, Ahnen DJ, Meester RGS, Barzi A and Jemal A: Colorectal cancer statistics, 2017. CA Cancer J Clin 67: 177-193, 2017.

6. Passiglia F, Bronte G, Bazan V, Galvano A, Vincenzi B and Russo A: Can KRAS and BRAF mutations limit the benefit of liver resection in metastatic colorectal cancer patients? A systematic review and meta-analysis. Crit Rev Oncol Hematol 99: 150-157, 2016.

7. Walther A, Johnstone E, Swanton C, Midgley R, Tomlinson I and Kerr D: Genetic prognostic and predictive markers in colorectal cancer. Nat Rev Cancer 9: 489-499, 2009.

8. Kaelin WG Jr: The concept of synthetic lethality in the context of anticancer therapy. Nat Rev Cancer 5: 689-698, 2005

9. Yan X, Hu Z, Feng Y, Hu X, Yuan J, Zhao SD, Zhang Y, Yang L, Shan W, He Q, et al: Comprehensive genomic characterization of long non-coding RNAs across human cancers. Cancer Cell 28 : 529-540, 2015

10. Mattick JS and Rinn JL: Discovery and annotation of long noncoding RNAs. Nat Struct Mol Biol 22: 5-7, 2015.

11. Talyan S, Andrade-Navarro MA and Muro EM: Identification of transcribed protein coding sequence remnants within lincRNAs. Nucleic Acids Res 46: 8720-8729, 2018.

12. Ponting CP, Oliver PL and Reik W: Evolution and functions of long noncoding RNAs. Cell 136: 629-641, 2009.

13. Bhan A, Soleimani M and Mandal SS: Long noncoding RNA and cancer: A new paradigm. Cancer Res 77: 3965-3981, 2017.

14. Wang L, Cho KB, Li Y, Tao G, Xie Z and Guo B: Long noncoding RNA (lncRNA)-mediated competing endogenous rna networks provide novel potential biomarkers and therapeutic targets for colorectal cancer. Int J Mol Sci 20: 5758, 2019.

15. Qi X, Zhang DH, Wu N, Xiao JH, Wang X and Ma W: ceRNA in cancer: Possible functions and clinical implications. J Med Genet 52: 710-718, 2015.

16. Wang Y, Hou J, He D, Sun M, Zhang P, Yu Y and Chen Y: The emerging function and mechanism of ceRNAs in cancer. Trends Genet 32: 211-224, 2016.

17. Song YX, Sun JX, Zhao JH, Yang YC, Shi JX, Wu ZH, Chen XW, Gao P, Miao ZF and Wang ZN: Non-coding RNAs participate in the regulatory network of CLDN4 via ceRNA mediated miRNA evasion. Nat Commun 8: 289, 2017.

18. Wong CC, Qian Y, Li X, Xu J, Kang W, Tong JH, To KF, Jin Y, $\mathrm{Li}$ W. Chen $\mathrm{H}$, et al: SLC25A22 promotes proliferation and survival of colorectal cancer cells With KRAS mutations and xenograft tumor progression in mice via intracellular synthesis of aspartate. Gastroenterology 151: 945-960.e6, 2016.

19. Song Y, Chen QT and He QQ: Identification of key transcription factors in endometrial cancer by systems bioinformatics analysis. J Cell Biochem 120: 15443-15454, 2019.

20. Reshef DN, Reshef YA, Finucane HK, Grossman SR, McVean G, Turnbaugh PJ, Lander ES, Mitzenmacher M and Sabeti PC: Detecting novel associations in large data sets. Science 334: 1518-1524, 2011

21. Tripathi S, Pohl MO, Zhou Y, Rodriguez-Frandsen A, Wang G, Stein DA, Moulton HM, DeJesus P, Che J, Mulder LC, et al: Meta- and orthogonal integration of influenza 'OMICs' data defines a role for UBR4 in virus budding. Cell Host Microbe 18: 723-735, 2015.

22. Zheng Y, Fang YC and Li J: PD-L1 expression levels on tumor cells affect their immunosuppressive activity. Oncol Lett 18 5399-5407, 2019.

23. Tai P, Yu E, Cserni G, Vlastos G, Royce M, Kunkler I and Vinh-Hung V: Minimum follow-up time required for the estimation of statistical cure of cancer patients: Verification using data from 42 cancer sites in the SEER database. BMC Cancer 5: 48, 2005.
24. Afrăsânie VA, Marinca MV, Alexa-Stratulat T, Gafton B, Păduraru M, Adavidoaiei AM, Miron L and Rusu C: KRAS, NRAS, BRAF, HER2 and microsatellite instability in metastatic colorectal cancer-practical implications for the clinician. Radiol Oncol 53: 265-274, 2019.

25. Cicenas J, Tamosaitis L, Kvederaviciute K, Tarvydas R, Staniute G, Kalyan K, Meskinyte-Kausiliene E, Stankevicius V and Valius M: KRAS, NRAS and BRAF mutations in colorectal cancer and melanoma. Med Oncol 34: 26, 2017.

26. Porru M, Pompili L, Caruso C, Biroccio A and Leonetti C: Targeting KRAS in metastatic colorectal cancer: Current strategies and emerging opportunities. J Exp Clin Cancer Res 37: 57, 2018

27. Li DS, Ainiwaer JL, Sheyhiding I, Zhang Z and Zhang LW: Identification of key long non-coding RNAs as competing endogenous RNAs for miRNA-mRNA in lung adenocarcinoma. Eur Rev Med Pharmacol Sci 20: 2285-2295, 2016.

28. Liu J, Kruswick A, Dang H, Tran AD, Kwon SM, Wang XW and Oberdoerffer P: Ubiquitin-specific protease 21 stabilizes BRCA2 to control DNA repair and tumor growth. Nat Commun 8: 137, 2017.

29. Cui K, Liu C, Li X, Zhang Q and Li Y: Comprehensive characterization of the rRNA metabolism-related genes in human cancer. Oncogene 39: 786-800, 2020

30. Li X, Li B, Ran P and Wang L: Identification of ceRNA network based on a RNA-seq shows prognostic lncRNA biomarkers in human lung adenocarcinoma. Oncol Lett 16: 5697-5708, 2018.

31. Xu XT, Xu Q, Tong JL, Zhu MM, Nie F, Chen X, Xiao SD and Ran ZH: MicroRNA expression profiling identifies miR-328 regulates cancer stem cell-like SP cells in colorectal cancer. Br J Cancer 106: 1320-1330, 2012

32. Shen K, Liang Q, Xu K, Cui D, Jiang L, Yin P, Lu Y, Li Q and Liu J: MiR-139 inhibits invasion and metastasis of colorectal cancer by targeting the type I insulin-like growth factor receptor. Biochem Pharmacol 84: 320-330, 2012.

33. Guo H, Hu X, Ge S, Qian G and Zhang J: Regulation of RAP1B by miR-139 suppresses human colorectal carcinoma cell proliferation. Int J Biochem Cell Biol 44: 1465-1472, 2012.

34. Wang F, Ma YL, Zhang P, Shen TY, Shi CZ, Yang YZ, Moyer MP, Zhang HZ, Chen HQ, Liang Y and Qin HL: SP1 mediates the link between methylation of the tumour suppressor miR-149 and outcome in colorectal cancer. J Pathol 229: 12-24, 2013.

35. Liu C, Kelnar K, Vlassov AV, Brown D, Wang J and Tang DG: Distinct microRNA expression profiles in prostate cancer stem/progenitor cells and tumor-suppressive functions of let-7. Cancer Res 72: 3393-3404, 2012.

36. Lulla AR, Slifker MJ, Zhou Y, Lev A, Einarson MB, Dicker DT and El-Deiry WS: miR-6883 family miRNAs target CDK4/6 to induce $\mathrm{G}_{1}$ phase cell-cycle arrest in colon cancer cells. Cancer Res 77: 6902-6913, 2017.

37. Yang R, Wei M, Yang F, Sheng Y and Ji L: Diosbulbin B induced $\mathrm{G}_{2} / \mathrm{M}$ cell cycle arrest in hepatocytes by miRNA-186-3p and miRNA-378a-5p-mediated the decreased expression of CDK1. Toxicol Appl Pharmacol 357: 1-9, 2018.

38. Spiller F, Medina-Pritchard B, Abad MA, Wear MA, Molina O, Earnshaw WC and Jeyaprakash AA: Molecular basis for Cdk1-regulated timing of Mis18 complex assembly and CENP-A deposition. EMBO Rep 18: 894-905, 2017.

39. Lopez-Mejia IC, Lagarrigue S, Giralt A, Martinez-Carreres L, Zanou N, Denechaud PD, Castillo-Armengol J, Chavey C, Orpinell M, Delacuisine B, et al: CDK4 phosphorylates AMPK $\alpha 2$ to inhibit its activity and repress fatty acid oxidation. Mol Cell 68: 336-349.e6, 2017.

This work is licensed under a Creative Commons Attribution-NonCommercial-NoDerivatives 4.0 International (CC BY-NC-ND 4.0) License. 